\title{
ON AREOLAR MONOGENIC FUNCTIONS
}

\author{
MAXWELL O. READE
}

Let $f(z)=u(x, y)+i v(x, y), z=x+i y$, be a complex-valued function defined in the unit circle $D:|z|<1 . f(z)$ is said to be areolar monogenic in $D$ if and only if $u(x, y)$ and $v(x, y)$ (and hence $f(z)$ ) have continuous partial derivatives of the second order such that

$$
u_{x y}=-2^{-1}\left(v_{x x}-v_{y y}\right), \quad v_{x y}=2^{-1}\left(u_{x x}-u_{y y}\right)
$$

hold in $D[3] .{ }^{1}$ It is known that an areolar monogenic function has partial derivatives of all orders [3].

Whereas (1) is a differential characterization of areolar monogenic functions, it is the integral characterization contained in the following theorem that forms the basis for this note.

THEOREM A [3]. If $f(z)$ is continuous in $D$, then a necessary and suffcient condition that $f(z)$ be areolar monogenic in $D$ is that there exist a function $g(z)$, analytic in $D$, such that

$$
g(z)=\frac{1}{\pi r^{2} i} \int_{C(z: r)} f(\zeta) d \zeta, \quad \zeta=\xi+i \eta,
$$

holds for all circles $C(z ; r)$, with center $z$ and radius $r$, in $D$.

It should be noted that a symbol once introduced holds its meaning throughout the paper.

If $f(z)$ is continuous in $D$, then the right-hand member of (2) is a function of $z$ and $r$, defined for $z$ in the circle $D_{r}:|z|<1-r$, and for all $r$ such that $0<r<1$. Now if the definition

$$
G_{r}(z) \equiv \frac{1}{\pi r^{2} i} \int_{C_{(z: r)}} f(\zeta) d \zeta, \quad 0<r<1,|z|<1-r,
$$

is made, then the following is an extension of Theorem A.

Theorem 1. If $f(z)$ is continuous in $D$, then a necessary and suffcient condition that $f(z)$ be areolar monogenic in $D$ is that $G_{r}(z)$ be analytic in $D_{r}$, for all $r, 0<r<1$.

Necessity. This is precisely the necessity part of Theorem A.

Sufficiency. Suppose that in addition $f(z)$ has continuous partial

Presented to the Society, December 29, 1946; received by the editors June 17, 1946.

${ }^{1}$ Numbers in brackers refer to the bibliography at the end of the paper. 
derivatives of the first order in $D$. Then from (3) and Green's lemma it follows that

$$
G_{r}(z)=\frac{1}{\pi r^{2}} \iint_{D(z ; r)} G(\zeta) d \xi d \eta
$$

where

$$
G(z) \equiv U(x, y)+i V(x, y) \equiv\left(u_{x}-v_{y}\right)+i\left(u_{y}+v_{x}\right),
$$

and where $D(z ; r)$ is the closed circular disc with center at $z$ and radius $r$. Since $f(z)$ has continuous partial derivatives of the first order in $D$, it follows from (4) and (5) that $G_{r}(z) \rightarrow G(z)$, as $r \rightarrow 0$, on each closed subset of $D$. Hence, since $G_{r}(z)$ is analytic in $D_{r}$, for all $r$, it follows that $G(z)$ is analytic in $D$. Therefore $U(x, y)$ and $V(x, y)$ are conjugate harmonic functions such that (4) and the Gauss mean-value theorem for harmonic functions yield

$$
G_{r}(z)=\frac{1}{\pi r^{2}} \iint_{D(z ; r)} G(\zeta) d \xi d \eta=G(z)
$$

for all $z$ in $D_{r}, 0<r<1$.

It now follows from Theorem A that $f(z)$ is areolar monogenic in $D$.

Now if $f(z)$ is merely continuous in $D$, then for sufficiently small $\rho$, the mean-value function

$$
A(f ; z ; \rho) \equiv \frac{1}{\pi \rho^{2}} \iint_{D(z ; \rho)} f(\zeta) d \xi d \eta
$$

satisfies the hypotheses of this lemma in the circle $D_{\rho}$, for $0<\rho<1$; moreover, $A(f ; z ; \rho)$ has continuous partial derivatives of the first order in $D_{\rho}[1]$. Therefore by the preceding part of this proof, it follows that for all sufficiently small $\rho$, the function

$$
\frac{1}{\pi r^{2} i} \int_{C(z ; r)} A(f ; \zeta ; \rho) d \zeta, \quad 0<r+\rho<1,
$$

is analytic in $D_{r+p}$ and independent of $r$. But $A(f ; z ; \rho) \rightarrow f(z)$ as $\rho \rightarrow 0$, on each closed subset of $D[1]$. Hence it follows that for $0<r<1$, the right-hand member of (2) is analytic and independent of $r$ in $D_{r}$. Hence, by Theorem $\mathrm{A}, f(z)$ is areolar monogenic in $D$.

CoRollary 1. If $f(z)$ is continuous in $D$, then a necessary and sufficient condition that $f(z)$ be areolar monogenic in $D$ is that $A(f ; z ; \rho)$ be areolar monogenic in $D_{\rho}$, for all $\rho, 0<\rho<1$.

CoRollary 2. If $f(z)$ is areolar monogenic in $D$, then $f(z)$ has the following mean-value property: 


$$
\int_{C(z ; r)} f(\zeta) d \zeta=\int_{C(z ; r)} A(f ; \zeta ; \rho) d \zeta
$$

for each $C(z ; r)$ in $D_{\rho}$.

Proofs of the corollaries are contained in the proof of Theorem 1.

The equation (2) and recent results on the polygonal mean-values of harmonic polynomials [2] suggest the analogue of (2) wherein $C(z ; r)$ is replaced by a regular $n$-gon $p_{n}(z ; r ; \phi), n \geqq 3$. Let $P_{n}(z ; r ; \phi)$ denote the closed, finite region bounded by the regular $n$-gon $p_{n}(z ; r ; \phi)$ whose center is at $z$ and whose inscribed circle has radius $r$; $\phi$ denotes the angle from $R$ to $N,-\pi / n \leqq \phi<\pi / n$, where $R$ is the ray extending horizontally to the right from $z$ and $N$ is the exterior normal at the point where $R$ emerges from the polygon.

Here $n$ denotes a fixed, positive integer, $n \geqq 3$, and $\phi$ always denotes an (arbitrary) angle, $-\pi / n \leqq \phi<\pi / n$. For brevity, $p_{n}(z ; r ; \phi)$ will be denoted by $p(z ; r)$ and $P_{n}(z ; r ; \phi)$ will be denoted by $P(z ; r) ;|P|$ will denote the area of $P(z ; r)$.

The analogue of (2) referred to above is

$$
F_{r, \phi}(z) \equiv \frac{1}{|P| i} \int_{p(z ; r)} f(\zeta) d \zeta,
$$

which is defined on an open subset $D_{r, \phi}$ of $D$, for sufficiently small $r$.

The following result is comparable to the preceding theorem.

THEOREM 2. If $f(z)$ is continuous in $D$, then a necessary and sufficient condition that $f(z)$ be areolar monogenic in $D$ is that $F_{r, \phi}(z)$ be analytic in $D_{r, \phi}$ for each pair $r, \phi$.

Necessity. If $f(z)$ is areolar monogenic in $D$, then $f(z)$ has continuous partial derivatives (of all orders) in $D[3]$. Hence (7) and Green's lemma yield the following representation for $F_{r, \phi}(z)$ :

$$
F_{r, \phi}(z)=\frac{1}{|P|} \iint_{P(z ; r)} G(\zeta) d \xi d \eta,
$$

where, by (1) and (5), $G(z)$ is analytic in $D$. Now (8) shows that $F_{r, \phi}(z)$ is an integral mean of $G(z)$, so that $F_{r, \phi}(z)$ is analytic wherever defined, that is, in $D_{r, \phi}$.

Sufficiency. First suppose that $f(z)$ has continuous partial derivatives of the first order in $D$. Then it follows from (7) and Green's lemma that $F_{r, \phi}(z)$ can be written in the form (8). It now follows from (8) that $F_{r, \phi}(z) \rightarrow G(z)$, as $r \rightarrow 0$, on each closed subset of $D$. Since $F_{r, \phi}(z)$ is analytic in $D_{r, \phi}$, for all sufficiently small $r$, it follows that 
$G(z)$ is analytic in $D$. Hence, as in the proof of Theorem 1 , it follows that $f(z)$ is areolar monogenic in $D$.

The requirement that $f(z)$ have continuous partial derivatives of the first order in $D$ may be removed as in the proof of Theorem 1 . This completes the proof.

It should be noted that if $f(z)$ is an arbitrary areolar monogenic function (hence, if $G(z)$ is an arbitrary analytic function) in $D$, then $F_{r, \phi}(z)$ is analytic in $D_{r, \phi}$, though not necessarily independent of $r$. Indeed, if $F_{r, \phi}(z)$ is to be both analytic and independent of $r$, in $D_{r, \phi}$, then the following result holds.

THEOREM 3. If $f(z)$ is continuous in $D$, then a necessary and sufficient condition that $F_{r, \phi}(z)$ be both analytic and independent of $r$ in $D_{r, \phi}$, for fixed $\phi$, is that $f(z)$ be areolar monogenic in $D$, with the representation

$$
f(z) \equiv 2^{-1} \sum_{0}^{n-1} c_{k}\left(\bar{z} z^{k}-z^{k-1}\right)+\Psi_{y}+i \Psi_{x},
$$

where $\bar{z}=x-i y$, where the $c_{k}$ are arbitrary complex constants, and where $\Psi(x, y)$ is an arbitrary function harmonic in $D$.

To prove Theorem 3, the following lemma is needed.

LEMMA. If $f(z)$ is areolar monogenic in $D$, then a necessary and sufficient condition that $G(z)$ be a polynomial in $z$ of degree at most $(n-1)$ is that $f(z)$ have the representation (9).

Necessity. Let $G(z)$ have the representation

$$
G(z)=\sum_{0}^{n-1} c_{k} z^{k}
$$

Now Haskell has shown [3] that for areolar monogenic $f(z)$ there exist real functions $\mu(x, y), \nu(x, y), \Psi(x, y)$, with $\Psi(x, y)$ harmonic in $D$, such that

$$
f(z) \equiv\left(\mu_{x}+\nu_{y}\right)+i\left(\nu_{x}-\mu_{y}\right)+\Psi_{y}+i \Psi_{x},
$$

where

$$
\mu+i_{\nu} \equiv-\frac{1}{2 \pi} \iint_{D} \log \frac{1}{|z-\zeta|} G(\zeta) d \xi d \eta
$$

If the substitutions $\zeta=r e^{i \theta}, z=\rho e^{i \psi}$ are made in (10) and (12), then (12) yields 


$$
\begin{aligned}
\mu+i \nu=- & \frac{1}{2 \pi} \int_{0}^{\rho} \int_{0}^{2 \pi}\left[\log \frac{1}{\rho}+\sum_{1}^{\infty} \frac{r^{k}}{k \rho^{k}} \cos k(\theta-\psi)\right] \\
& \cdot\left[\sum_{0}^{n-1} c_{k} r^{k} e^{i k \theta}\right] r d r d \theta \\
- & \frac{1}{2 \pi} \int_{\rho}^{1} \int_{0}^{2 \pi}\left[\log \frac{1}{r}+\sum_{1}^{\infty} \frac{\rho^{k}}{k r^{k}} \cos k(\theta-\psi)\right] \\
& \cdot\left[\sum_{0}^{n-1} c_{k} r^{k} e^{i k \theta}\right] r d r d \theta \\
=\frac{1}{4} & {\left[\sum_{0}^{n-1} \frac{c_{k}}{k+1} \bar{z} z^{k+1}-\sum_{1}^{n-1} \frac{c_{k}}{k} z^{k}-c_{0}\right] . }
\end{aligned}
$$

The representation (9) for $f(z)$ now follows from (11) and (13).

Sufficiency. If $f(z)$ is given by (9), then a computation shows that $G(z)$, given by (5), has the form (10).

Proof of Theorem 3. If $f(z)$ is areolar monogenic in $D$, with representation (9), then it follows from the lemma that $G(z)$ has the form (10), such that $U(x, y)$ and $V(x, y)$ are harmonic polynomials of degree at most $(n-1)$. It is known that such harmonic polynomials satisfy

$$
\begin{aligned}
& U(x, y) \equiv U(z)=\frac{1}{|P|} \iint_{P(z ; r)} U(\zeta) d \xi d \eta, \\
& V(x, y) \equiv V(z)=\frac{1}{|P|} \iint_{P(z ; r)} V(\zeta) d \xi d \eta,
\end{aligned}
$$

for each $P(z ; r)$ in $D[2]$. From (5), (8) and (14), it follows that $F_{r, \phi}(z)$ is independent of $r, \phi$. This proves the necessity part of the theorem.

On the other hand, if $F_{r, \phi}(z)$ is analytic and independent of $r$ in $D_{r, \phi}$, then by the lemma, $f(z)$ is areolar monogenic in $D$. Moreover, it follows that the real and imaginary parts of $G(z)$ satisfy (14) and hence $U(x, y)$ and $V(x, y)$ have the representations implied by

$$
G(z) \equiv U+i V=\sum_{0}^{n-1} c_{k} z^{k}+c_{n} I\left(z_{k} \alpha\right), \quad \alpha=e^{i \psi},
$$

where the symbol " $I$ " means "the imaginary part of" [2]. However, since $U(x, y)$ and $V(x, y)$ are conjugate harmonic functions, it follows that $c_{n}=0$ in (15). Hence $G(z)$ is a polynomial of degree at most $(n-1)$; therefore, by the lemma, $f(z)$ is areolar monogenic in $D$ with representation (9). The proof is now complete. 
The author is indebted to the referee for the observation that Theorem 3 above is true for variable $\phi$.

\section{BIBLIOGRAPHY}

1. H. E. Bray, Proof of a formula for an area, Bull. Amer. Math. Soc. vol. 29 (1923) pp. 264-270.

2. E. F. Beckenbach and Maxwell Reade, Mean-values and harmonic polynomials, Trans. Amer. Math. Soc. vol. 53 (1943) pp. 230-238.

3. R. N. Haskell, Areolar monogenic functions, Bull. Amer. Math. Soc. vol. 52 (1946) pp. 332-337.

Purdue University 